\section{RSP}

http://www.rsp.fsp.usp.br/
Revista de Saúde Pública

\title{
Tendência da mortalidade infantil no município de Rio Branco, AC, 1999 a 2015
}

\author{
Alanderson Alves Ramalho', Andréia Moreira de Andrade', Fernanda Andrade Martins', \\ Rosalina Jorge Koifman" \\ ' Universidade Federal do Acre. Centro de Ciências da Saúde e do Desporto. Rio Branco, AC, Brasil \\ " Fundação Oswaldo Cruz. Escola Nacional de Saúde Pública. Rio de Janeiro, RJ, Brasil
}

\section{RESUMO}

OBJETIVO: Analisar a tendência da mortalidade infantil em Rio Branco, AC, de 1999 a 2015.

MÉTODOS: Estudo observacional ecológico de série temporal, em que foram utilizados dados de óbitos do Sistema de Informações sobre Mortalidade e Nascimentos do Sistema de Informações sobre Nascidos Vivos. Estimou-se a variação percentual anual por meio do software Joinpoint.

RESULTADOS: A taxa de mortalidade infantil reduziu de 26,99, em 1999, para 14,50, em 2015, por 1.000 nascidos vivos, com variação percentual anual de -4,37 (IC95\% -5,4- -3,4). Quando estratificada por componentes etários, o neonatal apresentou variação percentual anual de -4,73 (IC95\% -5,7--3,7), e o pós-neonatal de -3,7 (IC95\% -5,4- -2,0). A evitabilidade, as causas evitáveis e as causas mal definidas apresentaram tendência descendente em todo o período e as causas não claramente evitáveis apresentaram tendência ascendente até 2008. O grupo de causas que mais contribuiu para os óbitos infantis no período estudado foi o das afecções perinatais, seguido pelo das malformações, das doenças infecciosas e parasitárias, e do grupo das doenças respiratórias.

CONCLUSÕES: Apesar da tendência decrescente da taxa de mortalidade infantil na capital, quando comparada à de países desenvolvidos, mostra-se relativamente elevada.

DESCRITORES: Mortalidade Infantil, tendências. Causas de Morte. Qualidade da Assistência à Saúde. Indicadores Básicos de Saúde. Estudos de Séries Temporais.

\footnotetext{
Alanderson Alves Ramalho

Universidade Federal do Acre Centro de Ciências da Saúde

e do Desporto

BR 364, km 04 - Distrito Industrial 69915-900 Rio Branco, AC, Brasil

E-mail: alandersonalves@hotmail.com

Recebido: 30 dez 2016

Aprovado: 20 jun 2017

Como citar: Ramalho AA, Andrade AM, Martins FA, Koifman RJ. Tendência da mortalidade infantil no município de Rio Branco, AC, 1999 a 2015. Rev Saude Publica. 2018;52:33.

Copyright: Este é um artigo de acesso aberto distribuído sob os termos da Licença de Atribuição Creative Commons, que permite uso irrestrito, distribuição e reprodução em qualquer meio, desde que o autor e a fonte originais sejam creditados.
} 


\section{INTRODUÇÃO}

As condições de vida e saúde de uma população podem ser avaliadas por meio de diversos indicadores de saúde. A taxa de mortalidade infantil (TMI) é considerada um dos indicadores mais sensíveis em detectar mudanças. A busca pela redução da TMI em países subdesenvolvidos e em desenvolvimento faz parte da agenda governamental a nível global e representa um grande desafio para os serviços de saúde e a sociedade como um todo ${ }^{1}$.

A mortalidade do menor de um ano é comumente classificada de acordo com seus componentes: neonatal (óbitos ocorridos do nascimento até o $27^{\circ}$ dia de vida) e pós-neonatal (óbitos ocorridos do $28^{\circ}$ ao $364^{\circ}$ dia de vida).

A mortalidade neonatal é sensível a fatores endógenos ou biológicos relacionados à gestação e ao parto. Sua redução envolve maior complexidade e maior custo na prevenção desses óbitos relacionados a problemas genéticos, malformação do feto, gravidez tardia e complicações no parto e pós-parto ${ }^{2-4}$.

A mortalidade pós-neonatal é um indicador sensível aos fatores externos que influenciam a ocorrência de óbitos nessa faixa etária. Refletem as condições do meio ambiente, nutricionais e de bem-estar em que essa população está inserida. Neste componente da TMI, as ações governamentais, como saneamento básico, distribuição de renda e maior oferta dos serviços médicos, têm um maior impacto na sua redução, principalmente quando incluem as classes sociais menos favorecidas ${ }^{5,6}$.

A estimativa da taxa de mortalidade infantil mostrou tendência de declínio no mundo inteiro, apresentando redução de $49 \%$, passando de 23 para 10 óbitos por cada 1.000 nascidos vivos de 1990 a 20157. Nos últimos anos, a Europa apresentou maior redução (55\%), seguida pelas Américas (54\%). A menor redução (21\%) foi observada nos países africanos.

Na América do Sul, considerando o ranking das estimativas das melhores TMI para 2015, o Brasil fica atrás do Chile, Uruguai, Argentina, Venezuela, Peru e Colômbia; e à frente do Equador, Paraguai, Suriname, Bolívia e Guiana ${ }^{7}$. O Ministério da Saúde analisou a tendência da TMI no Brasil de 1998 a 2007 e observou declínio em todas as regiões ${ }^{8}$. No final do período analisado, os maiores valores das taxas foram registrados na região Nordeste (28,7/1.000 nascidos vivos [NV]), seguido das regiões Norte (22,1/1.000 NV), Centro-Oeste (16,5/1.000 NV), Sudeste $(14,6 / 1.000 \mathrm{NV})$ e Sul (12,9/1.000 NV). Esse ordenamento ficou inalterado no decorrer desta série temporal, indicando a manutenção das diferenças regionais existentes no país ${ }^{1}$.

Conhecer a evolução da TMI pode favorecer a tomada de decisões na condução das políticas públicas de saúde para garantir melhor assistência durante o pré-natal, parto e puerpério, esforços necessários para a diminuição da mortalidade infantil. Há escassez de publicações que utilizaram análise de séries temporais da mortalidade infantil na região Norte do Brasil. No estado do Acre, não foram encontrados estudos com essa metodologia. Desta forma, o objetivo deste estudo foi analisar a tendência da mortalidade infantil em Rio Branco, AC, de 1999 a 2015.

\section{MÉTODOS}

Estudo observacional descritivo ecológico de série temporal da mortalidade infantil em Rio Branco entre 1999 e 2015. Rio Branco, capital do Acre, tem 348.354 habitantes (45,9\% da população do Estado), distribuídos em uma área de $9.962 \mathrm{~km}^{2}$ (6,5\% do território do Estado), e cerca de $90 \%$ residem na área urbana9 .

As fontes de dados foram as estatísticas vitais do Departamento de Informática do Sistema Único de Saúde (Datasus). O número de óbitos de menores de um ano foi obtido do Sistema de Informações sobre Mortalidade (SIM) e o número de nascimentos do Sistema de Informações sobre Nascidos Vivos (Sinasc). 
Foram calculadas as TMI por três sistemas de categorização: componentes etários, evitabilidade e segundo os principais grupos de causa básica do óbito.

A TMI foi definida como a razão entre o número total de óbitos em menores de um ano no ano e no denominador o número total de nascidos vivos, expressa por 1.000 nascidos vivos. O componente neonatal precoce foi obtido pela razão do número de óbitos entre zero e seis dias de vida registrados no ano dividido pelo número total de nascidos vivos. O neonatal tardio correspondeu a essa mesma razão tendo o número de óbitos de sete a 27 dias no numerador e o componente pós-neonatal, o número de óbitos de 28 a 364 dias.

Para a classificação por evitabilidade, utilizou-se a Lista Brasileira de Mortes Evitáveis ${ }^{10}$, que considera causas de mortes mal definidas, causas evitáveis (reduzíveis pelas ações de imunização; reduzível pela adequada atenção à mulher na gestação e no parto e ao recém-nascido; reduzíveis por ações adequadas de diagnóstico e tratamento; e reduzíveis por ações de promoção da saúde vinculadas a ações de atenção em saúde); e causas não evitáveis (demais causas).

O registro da causa de óbito foi baseado na $10^{\mathrm{a}}$ Classificação Estatística Internacional de Doenças e Problemas Relacionados com a Saúde (CID-10). Os seguintes grupos foram definidos para a análise de causas de morte: afecções perinatais (CID-10 P00 a P96); malformações, deformidades e anomalias (CID-10 Q00 a Q99); doenças respiratórias (CID-10 J00 a J99); doenças infecciosas e parasitárias (CID-10 A00 a B99); e demais causas (todos os demais códigos do CID-10). Nesta categorização, as mortes por causas mal definidas nos menores de um ano foram redistribuídas proporcionalmente entre os grupos de causas analisados.

A análise da tendência foi realizada com as estimativas da variação percentual anual (Anual Percentage Change [APC]) e a variação percentual anual média (Average Anual Percentage Change [AAPC]) da taxa de mortalidade infantil de 1999 a 2015 por meio de regressão de Poisson. Utilizou-se o programa estatístico Joinpoint (http://surveillance.cancer.gov/ joinpoint/). A técnica de joinpoint utiliza as taxas log-transformadas para identificar os pontos de inflexão (joinpoints), ao longo do período, capazes de descrever uma mudança significativa na tendência por meio da $\mathrm{APC}^{11}$. Como os fenômenos biológicos nem sempre se comportam de maneira uniforme, uma taxa pode apresentar mudanças no ritmo de variação ao longo do tempo. Quando ocorre essa situação, a análise de segmentos pode representar melhor o fenômeno observado. No conceito da APC do segmento (APC), os pontos de inflexão, (joinpoint) correspondem a k-1 segmentos. A medida resumo dos diversos APC é a AAPC que corresponde à mudança percentual anual média. Nas situações em que apenas um APC compreende todo o período estudado, a AAPC corresponde à APC. Os testes de significância para escolha do melhor modelo basearam-se no método de permutação de Monte Carlo, considerando $\mathrm{p}<0,05$. Para minimizar o efeito de possíveis autocorrelações, utilizou-se a opção "fit an autocorrelated errors model based on the data" disponível no software.

\section{RESULTADOS}

Nasceram vivas 123.800 crianças em Rio Branco de 1999 a 2015. A média anual foi de 7.282,35 (DP =624,27; mín. =6.437; máx. =8.819). A tendência de nascidos vivos apresentou variação percentual anual de -2,29 (IC95\% -2,9- -1,7) até 2010. Posteriormente, a variação percentual anual foi de 1,64, todavia não houve evidências estatísticas para a variação de 2010 a 2015 (IC95\% -0,5-3,8).

O componente neonatal precoce foi o que mais contribuiu para a mortalidade infantil no período, com proporção média de 52,2\% (máx. = 62,2\% em 1999, mín. = 41,4\% em 2009); o componente neonatal tardio apresentou média de 14,3\% (máx. $=19,8 \%$ em 2009, mín. = 8,9\% em 2012); e o componente pós-neonatal apresentou média de 33,5\% (máx. = 37,8\% em 2006, mín. = 25,6\% em 1999). Quanto à evitabilidade, as causas evitáveis contribuíram com média de 66,2\% (mín. = 57,6\%; máx. = 72,4\%), as não claramente evitáveis obtiveram média de 27,2\% (mín. = 14,2\%; máx. = 37,6\%) e as causas mal definidas 6,6\% (mín. = 0,9\%; máx. $=13,4 \%)($ Tabela 1$)$. 
O grupo de causas básicas que mais contribuiu para os óbitos infantis no período foi o de afecções perinatais ( média $=57,8 \%$; mín. $=45,8 \%$; máx. $=69,2 \%$ ), seguido por malformações (média $=18,7 \%$; mín. $=10,9 \%$; máx. $=25,5 \%$ ), doenças infecciosas e parasitárias (média $=$ 6,9\%; mín. = 4,2\%; máx. = 12,2\%), e doenças respiratórias ( média = 5,3\%; mín. = 2,7\%; máx. = $7,4 \%$ ). As demais causas agrupadas contribuíram, em média, com 11,3\% dos óbitos infantis (mín. = 5,3\%; máx. = 18,8\%) $($ Tabela 1).

A TMI (por 1.000 nascidos vivos) reduziu no período analisado de 27,0 em 1999 para 14,5 em 2015. Essa redução ocorreu também quando estratificada por componentes (neonatal precoce e tardia; e pós-neonatal), critérios de evitabilidade, e grupos de causas (Tabela 1). Essa redução significativa da TMI entre 1999 e 2015, teve APC de -4,4 (IC95\% -5,4- -3,4) (Figura 1, Tabela 2).

Tabela 1. Taxa de mortalidade infantil por 1.000 nascidos vivos e mortalidade proporcional em menores de um ano (\%) por faixa etária, evitabilidade e causas de óbito. Rio Branco, AC, Brasil, 1999 a 2015.

\begin{tabular}{|c|c|c|c|c|c|c|c|c|c|c|c|c|c|c|c|}
\hline \multirow{3}{*}{ Ano } & \multirow{2}{*}{\multicolumn{2}{|c|}{$\begin{array}{l}\text { Frequência } \\
\text { absoluta }\end{array}$}} & \multicolumn{13}{|c|}{ Taxa de mortalidade infantil por mil nascidos vivos e mortalidade proporcional $(\%)$} \\
\hline & & & \multicolumn{5}{|c|}{ Componente etário } & \multicolumn{3}{|c|}{ Evitabilidade } & \multicolumn{5}{|c|}{ Grupo de causas } \\
\hline & $\begin{array}{l}\text { Nascidos } \\
\text { vivos }\end{array}$ & $\begin{array}{l}\text { Óbitos } \\
\text { infantis }\end{array}$ & $<1$ ano & Neonatal & NP & NT & PN & CE & NCE & MD & AP & MDA & DR & DIP & DC \\
\hline 1999 & 8.819 & 238 & 26,99 & 20,07 & $\begin{array}{c}16,78 \\
(62,2 \%)\end{array}$ & $\begin{array}{c}3,29 \\
(12,2 \%)\end{array}$ & $\begin{array}{c}6,92 \\
(25,6 \%)\end{array}$ & $\begin{array}{c}18,82 \\
(69,7 \%)\end{array}$ & $\begin{array}{c}5,22 \\
(19,3 \%)\end{array}$ & $\begin{array}{c}2,95 \\
(10,9 \%)\end{array}$ & $\begin{array}{c}17,44 \\
(64,6 \%)\end{array}$ & $\begin{array}{c}3,95 \\
(14,6 \%)\end{array}$ & $\begin{array}{c}1,27 \\
(4,7 \%)\end{array}$ & $\begin{array}{c}1,53 \\
(5,7 \%)\end{array}$ & $\begin{array}{c}2,8 \\
(10,4 \%)\end{array}$ \\
\hline 2000 & 8.287 & 232 & 28 & 18,22 & $\begin{array}{c}14,84 \\
(53,0 \%)\end{array}$ & $\begin{array}{c}3,38 \\
(12,1 \%)\end{array}$ & $\begin{array}{c}9,77 \\
(34,9 \%)\end{array}$ & $\begin{array}{c}20,27 \\
(72,4 \%)\end{array}$ & $\begin{array}{c}3,98 \\
(14,2 \%)\end{array}$ & $\begin{array}{c}3,74 \\
(13,4 \%)\end{array}$ & $\begin{array}{c}19,36 \\
(69,2 \%)\end{array}$ & $\begin{array}{c}3,06 \\
(10,9 \%)\end{array}$ & $\begin{array}{c}1,67 \\
(6,0 \%)\end{array}$ & $\begin{array}{c}1,95 \\
(7,0 \%)\end{array}$ & $\begin{array}{c}1,95 \\
(7,0 \%)\end{array}$ \\
\hline 2001 & 7.788 & 184 & 23,63 & 16,05 & $\begin{array}{c}13,87 \\
(58,7 \%)\end{array}$ & $\begin{array}{c}2,18 \\
(9,2 \%)\end{array}$ & $\begin{array}{c}7,58 \\
(32,1 \%)\end{array}$ & $\begin{array}{c}16,95 \\
(71,7 \%)\end{array}$ & $\begin{array}{c}4,88 \\
(20,7 \%)\end{array}$ & $\begin{array}{c}1,8 \\
(7,6 \%)\end{array}$ & $\begin{array}{c}15,29 \\
(64,7 \%)\end{array}$ & $\begin{array}{c}3,89 \\
(16,5 \%)\end{array}$ & $\begin{array}{c}1,39 \\
(5,9 \%)\end{array}$ & $\begin{array}{c}1,81 \\
(7,6 \%)\end{array}$ & $\begin{array}{c}1,25 \\
(5,3 \%)\end{array}$ \\
\hline 2002 & 7.710 & 161 & 20,88 & 14,79 & $\begin{array}{c}12,06 \\
(57,8 \%)\end{array}$ & $\begin{array}{c}2,72 \\
(13,0 \%)\end{array}$ & $\begin{array}{c}6,10 \\
(29,2 \%)\end{array}$ & $\begin{array}{c}14,66 \\
(70,2 \%)\end{array}$ & $\begin{array}{c}4,41 \\
(21,1 \%\end{array}$ & $\begin{array}{c}1,82 \\
(8,7 \%)\end{array}$ & $\begin{array}{c}13,78 \\
(66,0 \%)\end{array}$ & $\begin{array}{c}3,41 \\
(16,3 \%)\end{array}$ & $\begin{array}{c}0,57 \\
(2,7 \%)\end{array}$ & $\begin{array}{c}1,28 \\
(6,1 \%)\end{array}$ & $\begin{array}{c}1,85 \\
(8,8 \%)\end{array}$ \\
\hline 2003 & 7.669 & 171 & 22,3 & 15,52 & $\begin{array}{c}12,52 \\
(56,1 \%)\end{array}$ & $\begin{array}{c}3,00 \\
(13,5 \%)\end{array}$ & $\begin{array}{c}6,78 \\
(30,4 \%)\end{array}$ & $\begin{array}{c}15,39 \\
(69,0 \%)\end{array}$ & $\begin{array}{c}5,09 \\
(22,8 \%)\end{array}$ & $\begin{array}{c}1,83 \\
(8,2 \%)\end{array}$ & $\begin{array}{c}13,63 \\
(61,1 \%)\end{array}$ & $\begin{array}{c}3,83 \\
(17,2 \%)\end{array}$ & $\begin{array}{c}0,85 \\
(3,8 \%)\end{array}$ & $\begin{array}{c}1,7 \\
(7,6 \%)\end{array}$ & $\begin{array}{c}2,27 \\
(10,2 \%)\end{array}$ \\
\hline 2004 & 7.259 & 164 & 22,59 & 14,74 & $\begin{array}{c}12,12 \\
(53,7 \%)\end{array}$ & $\begin{array}{c}2,62 \\
(11,6 \%)\end{array}$ & $\begin{array}{c}7,85 \\
(34,8 \%)\end{array}$ & $\begin{array}{c}14,19 \\
(62,8 \%)\end{array}$ & $\begin{array}{c}5,37 \\
(23,8 \%)\end{array}$ & $\begin{array}{c}3,03 \\
(13,4 \%)\end{array}$ & $\begin{array}{c}14,00 \\
(62,0 \%)\end{array}$ & $\begin{array}{c}4,77 \\
(21,1 \%)\end{array}$ & $\begin{array}{c}1,27 \\
(5,6 \%)\end{array}$ & $\begin{array}{c}1,27 \\
(5,6 \%)\end{array}$ & $\begin{array}{c}1,27 \\
(5,6 \%)\end{array}$ \\
\hline 2005 & 7.288 & 149 & 20,44 & 12,49 & $\begin{array}{c}10,29 \\
(50,3 \%)\end{array}$ & $\begin{array}{c}2,20 \\
(10,7 \%)\end{array}$ & $\begin{array}{c}7,96 \\
(38,9 \%)\end{array}$ & $\begin{array}{c}12,62 \\
(61,7 \%)\end{array}$ & $\begin{array}{c}6,45 \\
(31,5 \%)\end{array}$ & $\begin{array}{c}1,37 \\
(6,7 \%)\end{array}$ & $\begin{array}{c}10,74 \\
(52,5 \%)\end{array}$ & $\begin{array}{c}4,71 \\
(23,0 \%)\end{array}$ & $\begin{array}{c}1,03 \\
(5,0 \%)\end{array}$ & $\begin{array}{c}2,21 \\
(10,8 \%)\end{array}$ & $\begin{array}{c}1,76 \\
(8,6 \%)\end{array}$ \\
\hline 2006 & 7.345 & 135 & 18,38 & 11,44 & $\begin{array}{c}9,39 \\
(51,1 \%)\end{array}$ & $\begin{array}{c}2,04 \\
(11,1 \%)\end{array}$ & $\begin{array}{c}6,94 \\
(37,8 \%)\end{array}$ & $\begin{array}{c}12,8 \\
(69,6 \%)\end{array}$ & $\begin{array}{c}5,17 \\
(28,1 \%)\end{array}$ & $\begin{array}{c}0,41 \\
(2,2 \%)\end{array}$ & $\begin{array}{c}9,33 \\
(50,8 \%)\end{array}$ & $\begin{array}{c}3,34 \\
(18,2 \%)\end{array}$ & $\begin{array}{c}0,97 \\
(5,3 \%)\end{array}$ & $\begin{array}{c}2,23 \\
(12,1 \%)\end{array}$ & $\begin{array}{c}2,51 \\
(13,6 \%)\end{array}$ \\
\hline 2007 & 7.067 & 155 & 21,93 & 14,57 & $\begin{array}{c}11,89 \\
(54,2 \%)\end{array}$ & $\begin{array}{c}2,69 \\
(12,3 \%)\end{array}$ & $\begin{array}{c}7,36 \\
(33,5 \%)\end{array}$ & $\begin{array}{c}13,58 \\
(61,9 \%)\end{array}$ & $\begin{array}{c}7,5 \\
(34,2 \%)\end{array}$ & $\begin{array}{c}0,85 \\
(3,9 \%)\end{array}$ & $\begin{array}{c}10,75 \\
(49,0 \%)\end{array}$ & $\begin{array}{c}5,59 \\
(25,5 \%)\end{array}$ & $\begin{array}{c}1,62 \\
(7,4 \%)\end{array}$ & $\begin{array}{c}1,03 \\
(4,7 \%)\end{array}$ & $\begin{array}{c}2,94 \\
(13,4 \%)\end{array}$ \\
\hline 2008 & 7.068 & 125 & 17,69 & 11,18 & $\begin{array}{c}8,21 \\
(46,4 \%)\end{array}$ & $\begin{array}{c}2,97 \\
(16,8 \%)\end{array}$ & $\begin{array}{c}6,51 \\
(36,8 \%)\end{array}$ & $\begin{array}{c}10,19 \\
(57,6 \%)\end{array}$ & $\begin{array}{c}6,51 \\
(36,8 \%)\end{array}$ & $\begin{array}{c}0,99 \\
(5,6 \%)\end{array}$ & $\begin{array}{c}8,09 \\
(45,8 \%)\end{array}$ & $\begin{array}{c}4,5 \\
(25,4 \%)\end{array}$ & $\begin{array}{c}0,6 \\
(3,4 \%)\end{array}$ & $\begin{array}{c}1,35 \\
(7,6 \%)\end{array}$ & $\begin{array}{c}3,15 \\
(17,8 \%)\end{array}$ \\
\hline 2009 & 6.538 & 116 & 17,74 & 10,86 & $\begin{array}{c}7,34 \\
(41,7 \%)\end{array}$ & $\begin{array}{c}3,52 \\
(20,0 \%)\end{array}$ & $\begin{array}{c}6,73 \\
(38,3 \%)\end{array}$ & $\begin{array}{c}11,47 \\
(64,7 \%)\end{array}$ & $\begin{array}{c}5,66 \\
(31,9 \%)\end{array}$ & $\begin{array}{c}0,61 \\
(3,4 \%)\end{array}$ & $\begin{array}{c}9,19 \\
(51,8 \%)\end{array}$ & $\begin{array}{c}2,85 \\
(16,1 \%)\end{array}$ & $\begin{array}{c}1,11 \\
(6,3 \%)\end{array}$ & $\begin{array}{c}1,27 \\
(7,1 \%)\end{array}$ & $\begin{array}{c}3,33 \\
(18,8 \%)\end{array}$ \\
\hline 2010 & 6.437 & 117 & 18,18 & 11,96 & $\begin{array}{c}8,54 \\
(47,0 \%)\end{array}$ & $\begin{array}{c}3,42 \\
(18,8 \%)\end{array}$ & $\begin{array}{c}6,21 \\
(34,2 \%)\end{array}$ & $\begin{array}{c}11,19 \\
(61,5 \%)\end{array}$ & $\begin{array}{c}5,13 \\
(28,2 \%)\end{array}$ & $\begin{array}{c}1,86 \\
(10,3 \%)\end{array}$ & $\begin{array}{c}10,91 \\
(60,0 \%)\end{array}$ & $\begin{array}{c}3,29 \\
(18,1 \%)\end{array}$ & $\begin{array}{c}0,52 \\
(2,9 \%)\end{array}$ & $\begin{array}{c}1,38 \\
(7,6 \%)\end{array}$ & $\begin{array}{c}2,08 \\
(11,4 \%)\end{array}$ \\
\hline 2011 & 6.902 & 90 & 13,04 & 8,98 & $\begin{array}{c}7,53 \\
(57,8 \%)\end{array}$ & $\begin{array}{c}1,45 \\
(11,1 \%)\end{array}$ & $\begin{array}{c}4,06 \\
(31,1 \%)\end{array}$ & $\begin{array}{c}8,98 \\
(68,9 \%)\end{array}$ & $\begin{array}{c}3,33 \\
(25,6 \%)\end{array}$ & $\begin{array}{c}0,72 \\
(5,6 \%)\end{array}$ & $\begin{array}{c}8,13 \\
(62,4 \%)\end{array}$ & $\begin{array}{c}1,84 \\
(14,1 \%)\end{array}$ & $\begin{array}{c}0,77 \\
(5,9 \%)\end{array}$ & $\begin{array}{c}0,77 \\
(5,9 \%)\end{array}$ & $\begin{array}{c}1,53 \\
(11,8 \%)\end{array}$ \\
\hline 2012 & 6.531 & 79 & 12,1 & 7,96 & $\begin{array}{c}6,89 \\
(57,0 \%)\end{array}$ & $\begin{array}{c}1,07 \\
(8,9 \%)\end{array}$ & $\begin{array}{c}4,13 \\
(34,2 \%)\end{array}$ & $\begin{array}{c}7,81 \\
(64,6 \%)\end{array}$ & $\begin{array}{c}3,22 \\
(26,6 \%)\end{array}$ & $\begin{array}{c}1,07 \\
(8,9 \%)\end{array}$ & $\begin{array}{c}6,89 \\
(56,9 \%)\end{array}$ & $\begin{array}{c}2,69 \\
(22,2 \%)\end{array}$ & $\begin{array}{c}0,84 \\
(6,9 \%)\end{array}$ & $\begin{array}{c}0,50 \\
(4,2 \%)\end{array}$ & $\begin{array}{c}1,18 \\
(9,7 \%)\end{array}$ \\
\hline 2013 & 7.116 & 95 & 13,35 & 9,42 & $\begin{array}{c}6,89 \\
(51,6 \%)\end{array}$ & $\begin{array}{c}2,53 \\
(18,9 \%)\end{array}$ & $\begin{array}{c}3,93 \\
(29,5 \%)\end{array}$ & $\begin{array}{c}9,56 \\
(71,6 \%)\end{array}$ & $\begin{array}{c}3,51 \\
(26,3 \%)\end{array}$ & $\begin{array}{c}0,28 \\
(2,1 \%)\end{array}$ & $\begin{array}{c}8,33 \\
(62,4 \%)\end{array}$ & $\begin{array}{c}2,15 \\
(16,1 \%)\end{array}$ & $\begin{array}{c}0,57 \\
(4,3 \%)\end{array}$ & $\begin{array}{c}0,57 \\
(4,3 \%)\end{array}$ & $\begin{array}{c}1,72 \\
(12,9 \%)\end{array}$ \\
\hline 2014 & 7.011 & 107 & 15,26 & 9,84 & $\begin{array}{c}6,56 \\
(43,0 \%)\end{array}$ & $\begin{array}{c}3,28 \\
(21,5 \%)\end{array}$ & $\begin{array}{c}5,42 \\
(35,5 \%)\end{array}$ & $\begin{array}{c}10,13 \\
(66,4 \%)\end{array}$ & $\begin{array}{c}4,99 \\
(32,7 \%)\end{array}$ & $\begin{array}{c}0,14 \\
(0,9 \%)\end{array}$ & $\begin{array}{c}7,77 \\
(50,9 \%)\end{array}$ & $\begin{array}{c}2,88 \\
(18,9 \%)\end{array}$ & $\begin{array}{c}1,15 \\
(7,5 \%)\end{array}$ & $\begin{array}{c}1,15 \\
(7,5 \%)\end{array}$ & $\begin{array}{c}2,30 \\
(15,1 \%)\end{array}$ \\
\hline 2015 & 6.965 & 101 & 14,50 & 9,76 & $\begin{array}{c}6,60 \\
(45,5 \%)\end{array}$ & $\begin{array}{c}3,16 \\
(21,8 \%)\end{array}$ & $\begin{array}{c}4,74 \\
(32,7 \%)\end{array}$ & $\begin{array}{c}8,9 \\
(61,4 \%)\end{array}$ & $\begin{array}{c}5,46 \\
(37,6 \%)\end{array}$ & $\begin{array}{c}0,14 \\
(1,0 \%)\end{array}$ & $\begin{array}{c}7,68 \\
(53,0 \%)\end{array}$ & $\begin{array}{c}3,33 \\
(23,0 \%)\end{array}$ & $\begin{array}{c}1,01 \\
(7,0 \%\end{array}$ & $\begin{array}{c}0,87 \\
(6,0 \%)\end{array}$ & $\begin{array}{c}1,59 \\
(11,0 \%)\end{array}$ \\
\hline
\end{tabular}

NP: neonatal precoce; NT: neonatal tardio; PN: pós-neonatal; CE: causas evitáveis; NCE: não claramente evitáveis; MD: mal definidas; AP: afecções perinatais; MDA: malformações, deformidades e anomalias; DR: doenças respiratórias; DIP: doenças infecciosas e parasitárias; DC: demais causas 

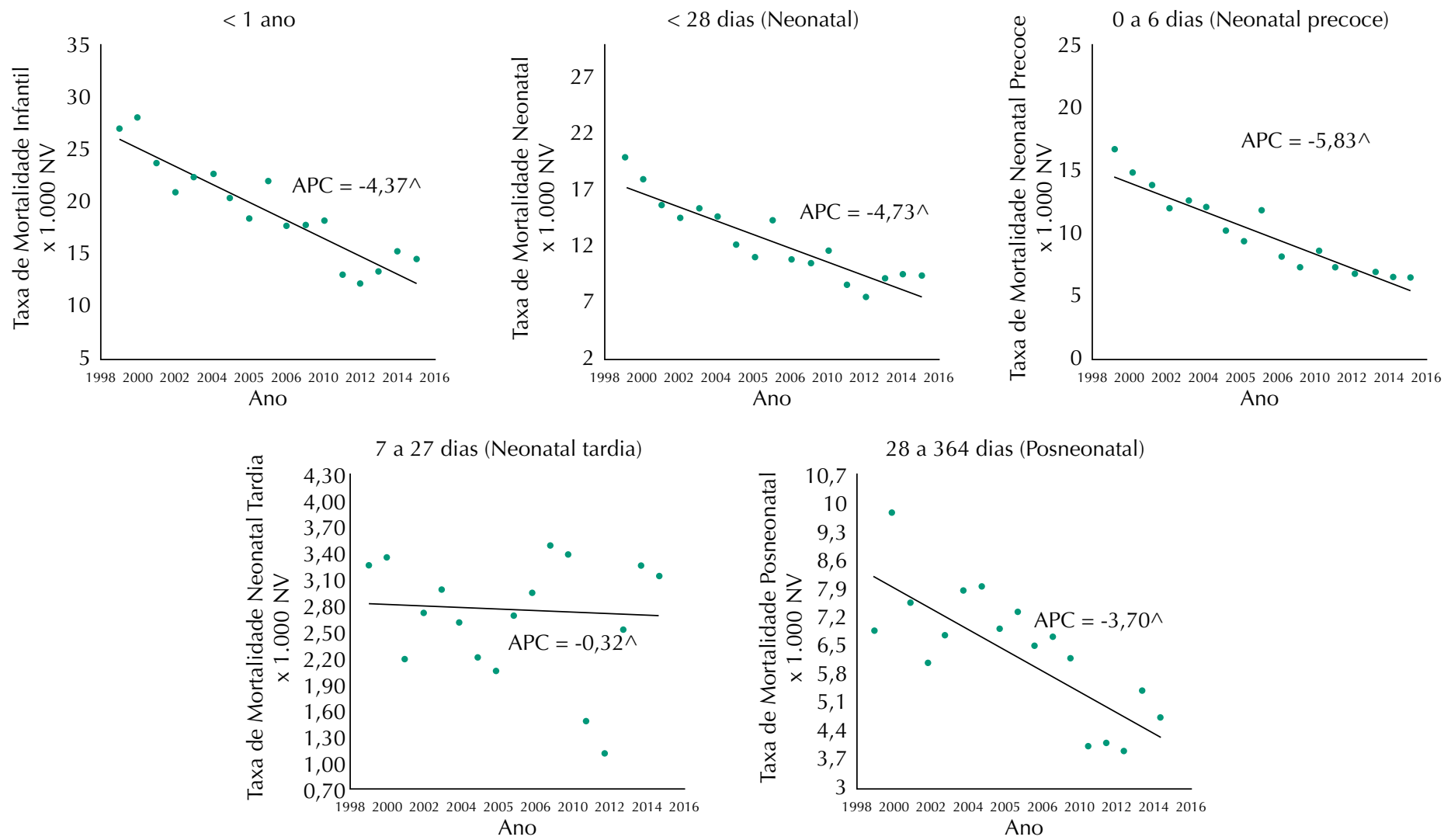

APC: variação percentual anual; NV: nascidos vivos

$\wedge$ estatisticamente diferente de zero.

Figura 1. Tendência das taxas de mortalidade infantil por componentes etários. Rio Branco, AC, Brasil, 1999 a 2015.

Tabela 2. Distribuição da variação percentual anual (APC) das taxas de mortalidade infantil por faixa etária, evitabilidade e causas de óbitos. Rio Branco, AC, Brasil, 1999 a 2015.

\begin{tabular}{|c|c|c|c|c|c|}
\hline \multirow{2}{*}{ Distribuição } & \multicolumn{2}{|c|}{ Taxa de mortalidade } & \multirow{2}{*}{ Óbitos } & \multirow{2}{*}{ VPA (IC95\%) } & \multirow{2}{*}{ Período } \\
\hline & 1999 & 2015 & & & \\
\hline$<1$ ano & 26,99 & 14,50 & 2.419 & $-4,37(-5,4--3,4)$ & 1999-2015 \\
\hline \multicolumn{6}{|l|}{ Componente etário } \\
\hline Neonatal & 20,07 & 9,76 & 1.616 & $-4,73(-5,7--3,7)$ & 1999-2015 \\
\hline NP & 16,78 & 6,60 & 1.283 & $-5,83(-6,7--4,9)$ & 1999-2015 \\
\hline NT & 3,29 & 3,16 & 333 & $-0,32(-3,0-2,4)$ & 1999-2015 \\
\hline Pós-neonatal & 6,92 & 4,74 & 802 & $-3,7(-5,4--2,0)$ & 1999-2015 \\
\hline \multicolumn{6}{|l|}{ Evitabilidade } \\
\hline CE & 18,82 & 8,90 & 1.615 & $-5,04(-6,0--4,1)$ & 1999-2015 \\
\hline \multirow[t]{3}{*}{ NCE } & 5,22 & 5,46 & 625 & $5,18(1,2-9,3)$ & 1999-2008 \\
\hline & & & & $-17,86(-35,3-4,4)$ & 2008-2012 \\
\hline & & & & $21,75(-4,0-54,5)$ & 2012-2015 \\
\hline MD & 2,95 & 0,14 & 179 & $-10,77(-15,5--5,7)$ & 1999-2015 \\
\hline \multicolumn{6}{|l|}{ Grupo de causas } \\
\hline AP & 17,44 & 7,68 & 1.314 & $-5,79(-7,0--4,6)$ & 1999-2015 \\
\hline \multirow[t]{3}{*}{ MDA } & 3,95 & 3,33 & 409 & $4,98(-0,9-11,2)$ & 1999-2007 \\
\hline & & & & $-18,33(-37,1-6,0)$ & 2007-2011 \\
\hline & & & & $9,30(-9,5-32,0)$ & 2011-2015 \\
\hline DR & 1,27 & 1,01 & 118 & $-2,83(-6,0-0,4)$ & 1999-2015 \\
\hline DIP & 1,53 & 0,87 & 157 & $-4,79(-8,0--1,5)$ & 1999-2015 \\
\hline \multirow[t]{4}{*}{$\mathrm{DC}$} & 2,80 & 1,59 & 242 & $-27,47(-66,2-55,8)$ & 1999-2001 \\
\hline & & & & $10,64(0,1-22,3)$ & 2001-2009 \\
\hline & & & & $-26,82(-69,4-75,2)$ & 2009-2012 \\
\hline & & & & $18,16(-24,7-85,4)$ & 2012-2015 \\
\hline
\end{tabular}

NP: neonatal precoce; NT: neonatal tardio; PN: pós-neonatal; CE: causas evitáveis; NCE: não claramente evitáveis; MD: mal definidas; AP: afecções perinatais; MDA: malformações, deformidades e anomalias; DR: doenças respiratórias; DIP: doenças infecciosas e parasitárias; DC: demais causas 
O componente neonatal apresentou APC de -4,7 (IC95\% -5,7- -3,7). Quando foi classificado em precoce e tardio, a redução ocorreu ao componente neonatal precoce (APC = -5,8; IC95\% $-6,7--4,9)$, e não existiu evidência estatística suficiente para afirmar variação no componente tardio. A mortalidade pós-neonatal apresentou tendência descendente significativa com APC de -3,7 (IC95\% -5,4- -2,0).

A mortalidade infantil por evitabilidade (Figura 2; Tabela 2) apresentou tendência descendente para todo o período no estrato de causas evitáveis, com APC de -5,0 (IC95\% -6,0- -4,1). As causas não claramente evitáveis apresentaram tendência ascendente até 2009 com APC de 5,2 (IC95\% 1,2-9,3).

As causas mal definidas apresentaram tendência descendente com APC de -10,8 (IC95\% $-15,5--5,7)$. As afecções perinatais apresentaram APC de -5,8 (IC95\% -7,0- -4,6); as doenças respiratórias APC de -2,8 (IC95\% -6,0-0,4) (Figura 3, Tabela 2). As malformações, deformidades e anomalias apresentaram variações cíclicas ao longo do período e não foi possível observar tendência com significância estatística.

As doenças infecciosas apresentaram tendência decrescente durante todo o período com APC de -4,8 (IC95\% -8,0- -1,5). As demais causas de óbito apresentaram tendência ascendente de 2001 a 2009, com APC de 10,64 (IC95\% 0,1-22,3). Não houve evidências estatísticas suficientes para afirmar variação percentual anual nos demais períodos (Figura 3, Tabela 2).

Causas evitáveis

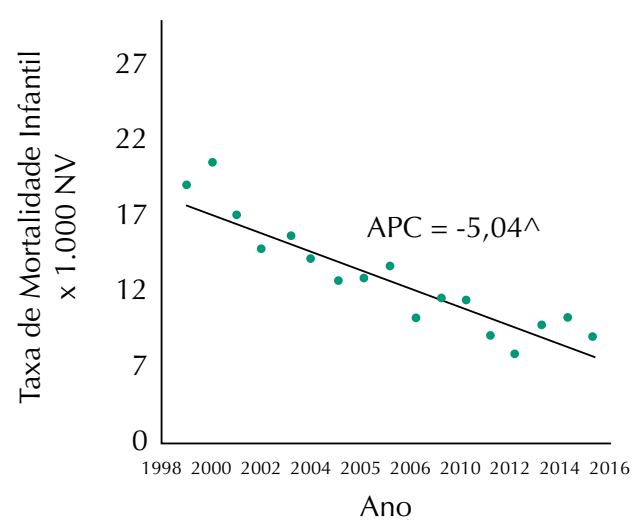

Causas não claramente evitáveis

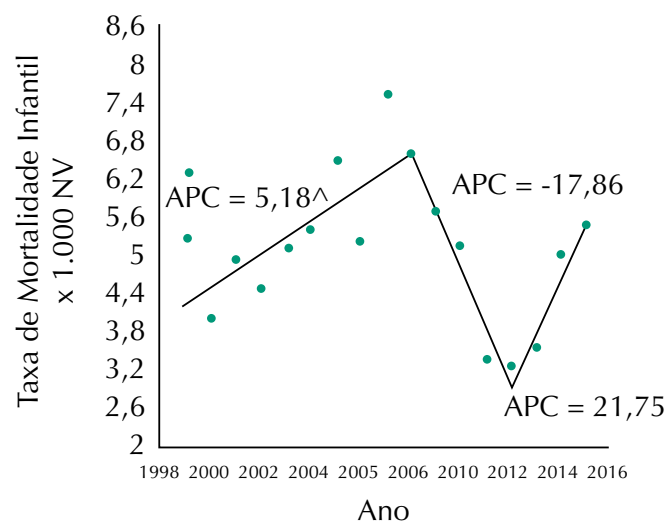

Causas mal definidas

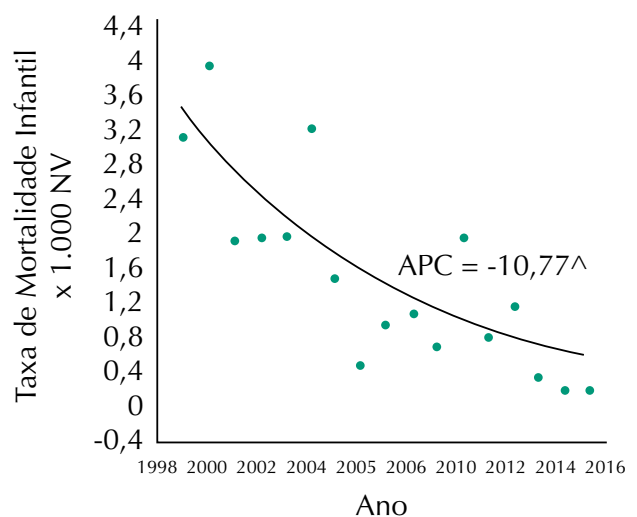

APC: variação percentual anual; NV: nascidos vivos $\wedge$ estatisticamente diferente de zero.

Figura 2. Tendência das taxas de mortalidade infantil por evitabilidade e causas mal definidas. Rio Branco, AC, Brasil, 1999 a 2015. 


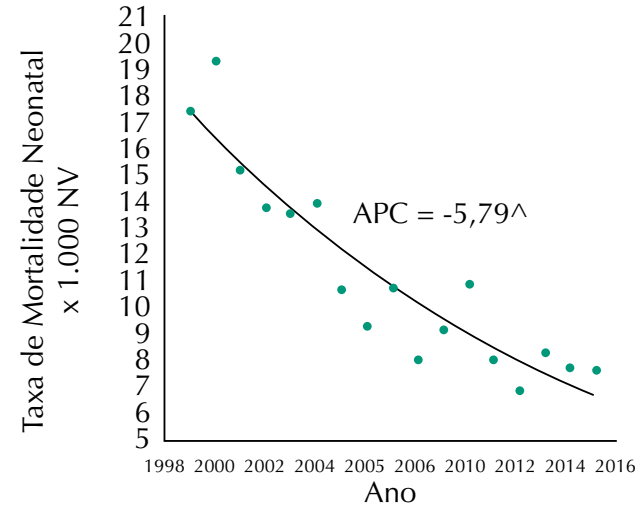

Doenças respiratórias

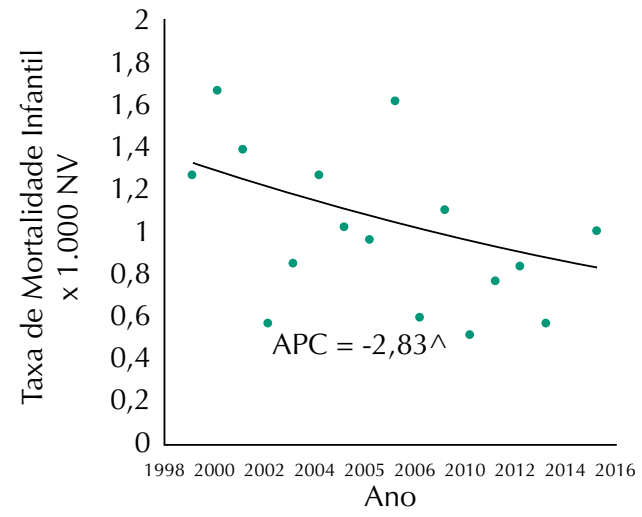

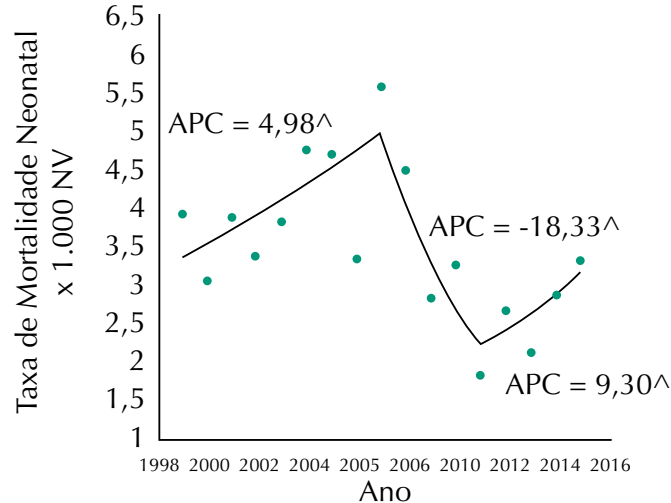

Doenças infecciosas e parasitárias

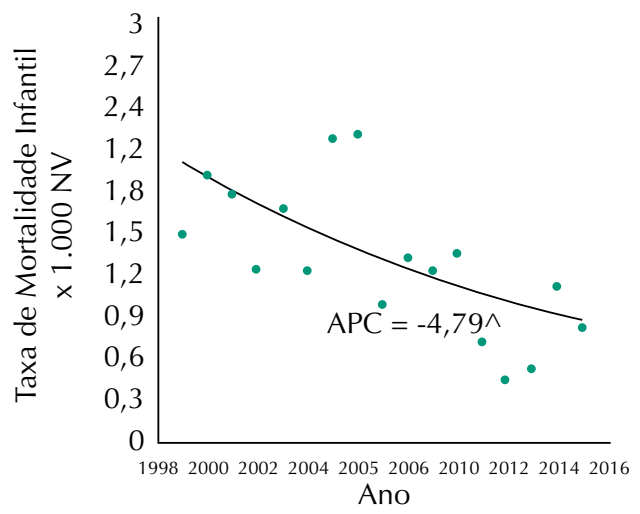

Demais causas

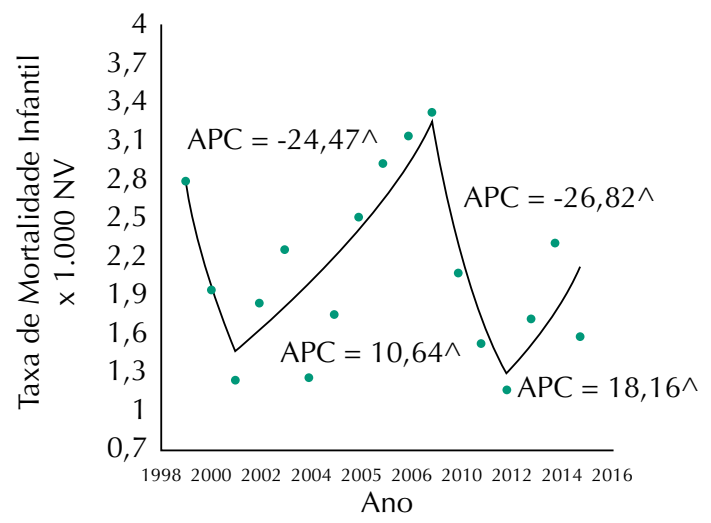

APC: variação percentual anual; NV: nascidos vivos $\wedge$ estatisticamente diferente de zero.

Figura 3. Tendência das taxas de mortalidade infantil por grupos de causas. Rio Branco, AC, Brasil, 1999 a 2015.

\section{DISCUSSÃO}

Neste estudo, a taxa de mortalidade infantil reduziu significativamente no período estudado. Quando estratificada por componentes etários, o neonatal apresentou variação percentual anual de -4,73 (IC95\% -5,7- -3,7) e o pós-neonatal, de -3,7 (IC95\% -5,4- -2,0). As causas evitáveis e as causas mal definidas apresentaram tendência descendente em todo o período e as causas não claramente evitáveis apresentaram tendência ascendente até 2008. O grupo de causas que mais contribuiu para os óbitos infantis no período estudado foi o das afecções perinatais, seguido pelos grupos das malformações, das doenças infecciosas e parasitárias, e o das doenças respiratórias. 
A redução mundial significativa na magnitude da mortalidade infantil ocorreu de forma importante na América Latina e no Caribe segundo relatório da Organização das Nações Unidas (ONU) em 2015, com um decréscimo estimado de 65,2\% (em 1990 de 46/1.000 NV; em 2015 de 16/1.000 NV). Nos países considerados desenvolvidos, a TMI passou de 13 para seis óbitos por 1.000 nascidos vivos no mesmo período?

Rio Branco acompanhou essa tendência do cenário mundial, com redução cumulativa na TMI de 46,3\% de 1999 a 2015. A TMI (14,5/1.000 NV) na capital acreana foi inferior à média nacional (15,02/1.000 NV) em 2015. No Brasil, entre 1990 e 2007, a TMI caiu de 47,1/1.000 NV em 1990 para 20,0/1.000 NV em 2007, com APC de -5,1. O declínio foi ligeiramente mais rápido na década de $1990(\mathrm{APC}=-5,5)$, depois a APC diminuiu para $-4,4$ na década de $2000^{12}$.

A queda da TMI ocorreu em todas as regiões brasileiras, com alterações mais marcantes no Nordeste, onde a APC foi de -5,9 (TMI = 75,8/1.000 NV em 1990 para 28,7/1.000 NV em 2007). Os declínios no Norte com APC de -4,3, Sudeste $(-4,9)$, Sul $(-4,5)$ e Centro-Oeste $(-4,1)$ também foram substanciais ${ }^{12}$.

Apesar da TMI no Brasil ser elevada quando comparada à de outros países com índice de desenvolvimento econômico similar ${ }^{13}$, foi suficiente para o Brasil atingir o quarto objetivo traçado no documento "Objetivos de Desenvolvimento do Milênio" ${ }^{14}$. Nele se assumiu como compromisso a redução nacional da taxa de mortalidade infantil para 15,7/1.000 NV até 2015 .

Quando a taxa de mortalidade infantil é muito baixa, ela é quase totalmente representada pela mortalidade neonatal. Quando é muito alta, observa-se exatamente o inverso, representada quase que na sua totalidade pela mortalidade pós-neonatal ${ }^{15}$.

O componente etário que mais contribuiu para o declínio da mortalidade infantil foi o neonatal precoce com APC de -5,83, seguido do pós-neonatal com APC de -3,7, em Rio Branco. O componente com menor contribuição foi o neonatal tardio $(-0,32)$.

Situação semelhante ocorreu em Guarulhos, SP, de 1996 a 2011, a APC da mortalidade neonatal precoce foi de -12,8 entre 1996 e 2002 e permaneceu estável até 2011. Já o componente pósneonatal apresentou APC de -5,7 para todo o período ${ }^{16}$.

Outros estudos observaram maior concentração de óbitos no componente neonatal precoce ${ }^{3,17}$, sugerindo causas de óbitos associadas principalmente à assistência ao pré-natal e parto, à atenção inadequada ao recém-nascido na sala de parto e à unidade neonatal, necessitando de maior atenção perinatal ${ }^{18-21}$.

No Distrito Federal, de 1990 a 2000, a APC do componente neonatal precoce foi de -4,76 e do pós-neonatal foi de $-7,80^{22}$. Em Porto Alegre, de 1996 a 2008, a APC do componente neonatal foi de $-3,5$ e a do pós-neonatal foi de $-4,1^{23}$.

As taxas de mortalidade neonatais também mostraram diminuição importante no Brasil entre 1990 e 2007. A APC foi de -3,2, menos acentuada que o declínio geral nas mortes infantis. Em contraste, a mortalidade pós-neonatal obteve APC de $-8,1^{12}$.

Mundialmente, a taxa de mortalidade neonatal reduziu de 36/1.000 NV em 1990 para 19/1.000 NV em 2015. Na América Latina e Caribe, nesse mesmo período, passou de 23/1.000 NV para 10/1.000 NV, e nos países desenvolvidos de 8/1.000 NV para 4/1.000 NV7.

Os grupos de causas que mais contribuíram para os óbitos infantis em Rio Branco no período foram o das afecções perinatais, seguido pelo das malformações, das doenças infecciosas e parasitárias, e o das doenças respiratórias.

O Ministério da Saúde, ao comparar óbitos infantis no Brasil em 2000, 2005, 2010 e 2012, observou que os fatores perinatais e maternos constituem as principais causas em menores de um ano, correspondendo a $52 \%$ dos óbitos infantis em 2012, seguido das malformações congênitas, responsáveis por 20,5\% dos óbitos infantis em $2012^{24}$. 
Em Aracajú, SE, as principais causas de óbito de 2001 a 2005 também foram afecções perinatais com TMI média de 18,1/1.000 NV com redução significativa para 12,3 de 2006 a 2010. As causas de mortalidade infantil por malformações congênitas e causas externas apresentaram aumento nos períodos ${ }^{25}$.

Segundo a pesquisa "Nascer no Brasil", os óbitos neonatais ocorreram principalmente devido à prematuridade, malformação congênita e infecções. As regiões Nordeste e Norte apresentaram a maior proporção de óbitos por infecção (26,9\% e 20,7\%). A maior proporção de óbitos por malformação congênita ocorreu no Sul e no Sudeste do Brasil ${ }^{26}$.

A redução da mortalidade no Brasil deve-se em sua maioria ao declínio dos óbitos evitáveis ${ }^{20,27,28}$. Segundo nota técnica do Ministério da Saúde entre 1996 e 2007, 70,0\% dos óbitos infantis poderiam ter sido evitados ${ }^{29}$. Uma análise das tendências dos óbitos infantis no Brasil entre 1997 e 2006 estimou redução de 37,0\% nas causas evitáveis, 75,7\% nas causas mal definidas e $2,2 \%$ nas demais causas de morte ${ }^{28}$.

As taxas de mortalidade por causas evitáveis tiveram redução de 52,7\% em Rio Branco entre 1999 e 2015 (APC = -5,04) e as causas mal definidas reduziram em 95,1\% no mesmo período $(\mathrm{APC}=-10,89)$. A expressiva redução da proporção dos óbitos classificados como causas mal definidas de morte em Rio Branco sugere maior acesso aos serviços de saúde.

A qualidade da informação de um sistema pode ser avaliada de acordo com a sua completude. No caso dos sistemas de informação de óbito, quando as causas mal definidas, não informadas ou ignoradas estão abaixo dos 10\%, considera-se uma informação de boa qualidade ${ }^{30}$.

A frequência média de causas mal definidas em Rio Branco neste estudo representou 6,6\% do total de óbitos infantis no período, caracterizando-se como uma informação de boa qualidade. Para a presente análise da tendência de mortalidade por causas evitáveis, assumiu-se que as causas mal definidas teriam distribuição proporcional semelhante às causas bem definidas.

Apesar da expressiva redução das causas evitáveis e mal definidas em Rio Branco, as causas não claramente evitáveis (demais causas) não apresentaram essa tendência. Como as causas evitáveis são sensíveis à atenção à saúde de boa qualidade, o Rio Branco, assim como todo o Brasil, pode estar experimentando melhoria no desempenho do sistema de saúde. Tal hipótese se sustenta no notório aprimoramento de oferta, cobertura e utilização das ações e serviços vinculados ao SUS ${ }^{8,24,28}$. Malta et al. ${ }^{28}$ ressaltam também que as causas não evitáveis tendem a declinar mais lentamente que as evitáveis.

A principal limitação deste estudo refere-se à utilização do método direto para o cálculo da mortalidade infantil, pois depende da cobertura e qualidade dos dados básicos de nascimentos e óbitos. A subnotificação de óbitos no país é um problema a ser enfrentado, especialmente nas regiões Norte e Nordeste. Isso faz com que o Ministério da Saúde recomende correções para locais cuja cobertura do Sinasc é inferior a $90 \%$ ou a $80 \%$ de um índice composto ${ }^{1}$. Todavia, em publicações recentes do Ministério da Saúde acerca da busca ativa de óbitos e nascimentos na Amazônia legal, o estado do Acre apresentou a maior cobertura no registro de óbitos e nascimentos da região Norte. Além disto, a cobertura da capital Rio Branco assemelha-se à de estados do Sul e Sudeste, justificando a utilização do método direto neste estudo.

A tendência da mortalidade infantil em Rio Branco acompanha o cenário brasileiro de redução. O componente etário que mais contribuiu para o declínio da taxa de mortalidade infantil foi o neonatal precoce, seguido do pós-neonatal. O componente neonatal tardio pouco contribuiu para a redução da mortalidade infantil na capital acreana no período analisado.

O grupo de causas que mais contribuiu para os óbitos infantis no período foi o das afecções perinatais, seguido pelo das malformações, das doenças infecciosas e parasitárias, e o das doenças respiratórias.

Quanto à evitabilidade, a TMI por causas evitáveis apresentou redução de 46,3\% entre 1997 e 2013, a TMI por causas mal definidas reduziu em 93,3\% no mesmo período. 
As expressivas reduções das causas mal definidas em Rio Branco sugerem maior acesso aos serviços de saúde, com possível melhora na atenção à saúde. Isso possibilita a identificação da causa de morte ou melhoria de preenchimento na declaração de óbito e gestão do SIM, buscando qualificar melhor as informações disponíveis. Todavia, a mortalidade infantil na capital é relativamente elevada quando comparada à apresentada em países desenvolvidos.

\section{REFERÊNCIAS}

1. Ministério da Saúde (BR), Secretaria de Vigilância em Saúde, Secretaria de Atenção à Saúde. Manual de vigilância do óbito infantil e fetal e do Comitê de Prevenção do Óbito Infantil e Fetal. 2.ed. Brasília (DF); 2009 [citado 10 out 2015]. (Série A. Normas e Manuais Técnicos). Disponível em: http://bvsms.saude.gov.br/bvs/publicacoes/manual_obito_infantil_fetal_2ed.pdf

2. França $E$, Lansky $S$. Mortalidade infantil neonatal no Brasil: situação, tendências e perspectivas. In: Rede Interagencial para a Saúde. Demografia e saúde: contribuição para análise de situação e tendências. Brasília (DF): Organização Pan-Americana da Saúde; 2009 [citado 10 out 2015]. p.83-112. (Série G. Estatística e Informação em Saúde) (Série Informe de Situação e Tendências). Disponível em: http://bvsms.saude.gov.br/bvs/publicacoes/demografia_saude_contribuicao_ tendencias.pdf

3. Kassar SB, Melo AM, Coutinho SB, Lima MC, Lira PI. Determinants of neonatal death with emphasis on health care during pregnancy, childbirth and reproductive history. J Pediatr (Rio J). 2013;89(3):269-77. https://doi.org/10.1016/j.jped.2012.11.005

4. Ribeiro VS, Silva AAM. Tendências da mortalidade neonatal em São Luís, Maranhão, Brasil, de 1979 a 1996. Cad Saude Publica. 2000;16(2):429-38. https://doi.org/10.1590/S0102-311X2000000200013

5. Ferrari RAP, Bertolozzi MR. Postnatal mortality in Brazilian territory: a literature review. Rev EsC Enferm USP. 2012;46(5):1207-14. https://doi.org/10.1590/S0080-62342012000500024

6. Tomé EA, Latorre MRDO. Tendências da mortalidade infantil no Município de Guarulhos: análise do período de 1971 a 1998. Rev Bras Epidemiol. 2001;4(3):153-67. https://doi.org/10.1590/S1415-790X2001000300003

7. United Nations Children's. Levels \& Trends in Child Mortality: report 2015. New York: UNICEF; 2015 [citado 10 out 2015]. Disponível em: http://www.unicef.org/publications/files/Child_ Mortality_Report_2015_Web_8_Sept_15.pdf

8. Ministério da Saúde (BR), Secretaria de Vigilância em Saúde. Saúde Brasil 2007: uma análise da situação de saúde. Brasília (DF); 2008 [citado 10 out 2015]. (Série G. Estatística e Informação em Saúde). Disponível em: http://bvsms.saude.gov.br/bvs/publicacoes/saude_brasil_2007.pdf

9. Instituto Brasileiro de Geografia e Estatística. Resultados do Censo 2010: Estado do Acre. Rio de Janeiro: IBGE; 2012 [citado 10 out 2015]. Disponível em: http://www.ibge.gov.br/home/ estatistica/populacao/censo2010/tabelas_pdf/total_populacao_acre.pdf

10. Malta DC, Duarte EC, Almeida MF, Dias MAS, Morais Neto OL, Moura L, et al. Lista de causas de mortes evitáveis por intervenções do Sistema Único de Saúde do Brasil. Epidemiol Serv Saude. 2007;16(4):233-44. https://doi.org/10.5123/S1679-49742007000400002

11. Kim HJ, Fay MP, Feuer EJ, Midthune DN. Permutation tests for joinpoint regression with applications to cancer rates. Stat Med. 2000;19(3):335-51. https://doi.org/10.1002/(SICI)1097-0258(20000215)19:3<335::AID-SIM336>3.0.CO;2-Z

12. Barros FC, Matijasevich A, Requejo JH, Giugliani E, Maranhão AG, Monteiro CA, et al. Recent trends in maternal, newborn, and child health in Brazil: progress toward Millennium Development Goals 4 and 5. Am J Public Health. 2010;100(10):1877-89. https://doi.org/10.2105/AJPH.2010.196816

13. Population Reference Bureau. World population data sheet 2012. Washington (DC): PRB; 2012 [citado 10 out 2015]. Disponível em: http://www.prb.org/Publications/Datasheets/2012/worldpopulation-data-sheet/data-sheet.aspx

14. Instituto de Pesquisa Econômica Aplicada; Secretaria de Planejamento e Investimentos Estratégicos. Objetivos de Desenvolvimento do Milênio (ODM): Relatório Nacional de Acompanhamento/Coordenação. Brasília (DF): IPEA; 2014 [citado 10 out 2015]. Disponível em: http://www.ipea.gov.br/portal/images/stories/PDFs/140523_relatorioodm.pdf 
15. Novaes HMD, Almeida MF, Ortiz LP. Redução da mortalidade infantil. 2.ed. São Paulo: BIREME/ OPAS/OMS; 2004 [citado 10 out 2015]. Disponível em: http://files.bvs.br/upload/M/2004/ Novaes_Reducao_mortalidade.pdf

16. Bando DH, Kawano MK, Kumagai LT, Gouveia JLV, Reis TM, Bernardo ES, et al. Tendência das taxas de mortalidade infantil e de seus componentes em GuarulhosSP, no período de 1996 a 2011. Epidemiol Serv Saude. 2014;23(4):767-72. https://doi.org/10.5123/S1679-49742014000400019

17. Nascimento RM, Leite AJM, Almeida NMGS, Almeida PC, Silva CF. [Determinants of neonatal mortality: a case-control study in Fortaleza, Ceará State, Brazil]. Cad Saude Publica. 2012;28(3):559-72. Portuguese. https://doi.org/10.1590/S0102-311X2012000300016

18. Almeida MFB, Guinsburg R, Martinez FE, Procianoy RS, Leone CR, Marba STM, et al. Perinatal factors associated with early deaths of preterm infants born in Brazilian Network on Neonatal Research centers. J Pediatr (Rio J). 2008;84(4):300-7. https://doi.org/10.1590/S0021-75572008000400004

19. Ferrari LSL, Brito ASJ, Carvalho ABR, Gonzáles MRC. Mortalidade neonatal no Município de Londrina, Paraná, Brasil, nos anos 1994, 1999 e 2002. Cad Saude Publica. 2006;22(5):1063-71. https://doi.org/10.1590/S0102-311X2006000500019

20. Lansky S, França E, Leal MC. Mortalidade perinatal e evitabilidade: revisão da literatura. Rev Saude Publica. 2002;36(6):759-72. https://doi.org/10.1590/S0034-89102002000700017

21. Mendes KG, Olinto MTA, Costa JSD. Case-control study on infant mortality in Southern Brazil. Rev Saude Publica. 2006;40(2):240-8. https://doi.org/10.1590/S0034-89102006000200009

22. Monteiro RA, Schmitz BAS. Infant mortality in the Federal District, Brazil: time trend and socioeconomic inequalities. Cad Saude Publica. 2007;23(4):767-74. https://doi.org/10.1590/S0102-311X2007000400004

23. Hernandez AR, Silva CH, Agranonik M, Quadros FM, Goldani MZ. Análise de tendências das taxas de mortalidade infantil e de seus fatores de risco na cidade de Porto Alegre, Rio Grande do Sul, Brasil, no período de 1996 a 2008. Cad Saude Publica. 2011;27(11):2188-96. https://doi.org/10.1590/S0102-311X2011001100012

24. Ministério da Saúde (BR), Secretaria de Vigilância em Saúde, Departamento de Análise de Situação em Saúde. Saúde Brasil 2013: uma análise da situação de saúde e das doenças transmissíveis relacionadas à pobreza. Brasília (DF); 2014. [citado 10 out 2015]. Disponível em: http://bvsms.saude.gov.br/bvs/publicacoes/saude_brasil_2013_analise_situacao_saude.pdf

25. Carvalho RAS, Santos VS, Melo CM, Gurgel RQ, Oliveira CCC. Inequalities in health: living conditions and infant mortality in Northeastern Brazil. Rev Saude Publica. 2015;49:5. https://doi.org/10.1590/S0034-8910.2015049004794

26. Lansky S, Friche AAL, Silva AAM, Campos D, Bittencourt SDA, Carvalho ML, et al. Pesquisa Nascer no Brasil: perfil da mortalidade neonatal e avaliação da assistência à gestante e ao recém-nascido. Cad Saude Publica. 2014;30 Supl 1:S192-207. https://doi.org/10.1590/0102-311X00133213

27. Duarte CMR. Reflexos das políticas de saúde sobre as tendências da mortalidade infantil no Brasil: revisão da literatura sobre a última década. Cad Saude Publica. 2007;23(7):1511-28. https://doi.org/10.1590/S0102-311X2007000700002

28. Malta DC, Duarte EC, Escalante JJC, Almeida MF, Sardinha LMV, Macário EM, et al. Mortes evitáveis em menores de um ano, Brasil, 1997 a 2006: contribuições para a avaliação de desempenho do Sistema Único de Saúde. Cad Saude Publica. 2010;26(3):481-91. https://doi.org/10.1590/S0102-311X2010000300006

29. Ministério da Saúde (BR), Secretaria de Vigilância em Saúde, Coordenação Geral de Informações e Análise Epidemiológica. Nota técnica: Vigilância Epidemiológica de Óbitos Infantis e Fetais. Brasília (DF); 2009 [citado 10 out 2015]. Disponível em: http://www.dive.sc.gov.br/conteudos/ Sistema_Informacao/Sim/Portarias/Nota_tecnica_Portaria_72_Vig_ob_infantil.pdf

30. Mello Jorge MHP, Gotlieb SLD, Soboll MLMS, Almeida MF, Latorre MRDO. Avaliação do Sistema de Informação sobre Nascidos Vivos e o uso de seus dados em epidemiologia e estatísticas de saúde. Rev Saude Publica. 1993;27 Supl:1-46. https://doi.org/10.1590/S0034-89101993000700001

Contribuição dos Autores: Concepção e planejamento do estudo: AAR, RJK. Coleta, análise e interpretação dos dados: AAR, AMA, FAM, RJK. Elaboração do manuscrito: AAR, RJK. Revisão do manuscrito: AAR, AMA, FAM, RJK. Aprovação da versão final: AAR, AMA, FAM, RJK. Responsabilidade pública pelo conteúdo do artigo: AAR.

Conflito de Interesses: Os autores declaram não haver conflito de interesses. 